\title{
HIGH ASPECT RATIO STRUCTURES ACHIEVED BY SACRIFICIAL CONFORMAL COATING
}

\author{
Florent Cros and Mark G. Allen \\ School of Electrical and Computer Engineering \\ Georgia Institute of Technology \\ Atlanta GA 30332-0250
}

\begin{abstract}
This paper reports on advances in the use of conformal scarificial layers to produce small gaps in high aspect ratio electroplated structures. The processes developed are based on the deposition and subsequent sacrificial removal of poly-(paraxylylene), or parylene, layers. Two processes are presented: one which allows released devices normal to the substrate surface and one which allows released devices parallel to the wafer surface. The two processes presented use conventional techniques of surface micromachining such as LIGA-like UV techniques, micromolding, and electroplating. Since the gaps between plated structures are defined not lithographically but instead by the thickness of the deposited parylene layer, and since the microstructures are fabricated in parylene-coated micromolds in a self-aligned fashion, extremely narrow gaps without the corresponding need for precise alignment and lithography through thick layers can be achieved. Examples of both processes, demonstrating 1:9 aspect ratios, are given.
\end{abstract}

\section{INTRODUCTION}

Thick metallic high aspect ratio microstructures present many advantages for both electrostatic and magnetic applications. For example, the driving voltages of electrostatic comb drives can be reduced by increasing the surface area (e.g., height) of the electrodes while decreasing the gap between electrodes. However, the level of processing difficulty increases with the above requirements. In addition to conventional thick photoresist processes, several different fabrication technologies have already been developed to realize high aspect ratio microstructures: LIGA (lithography, galvanoforming, and molding) [1], photosensitive polyimide [2] or epoxy [3] processes, and an electroplated aluminum sacrificial layer process [4]. Each of these processes has advantages and disadvantages which dictate their use in certain applications. The purpose of this paper is to describe the advantages which can be obtained by the combination of epoxy-based plating molds with a conformal parylene sacrificial layer. The idea is similar to that presented in the aluminum electroplating approach [4], but replaces the aluminum deposition with a parylene deposition. Since electrodeposited aluminum has the drawback that it should be electrodeposited from an ethereal solution (which is both water sensitive and highly flammable), and since parylene is a commonly used large area manufacturing deposition process without these drawbacks, it is anticipated that a parylene-based process will be able to be more widely implemented than an aluminum process, especially on larger area substrates.
Parylene (poly-(para-xylylene)) deposition provides an excellent conformal film with thickness ranging from 2 to 50 microns [5]. Moreover, it has excellent chemical resistance and can be deposited at low temperature $\left(25^{\circ} \mathrm{C}\right)$ so that thermal damage to any previous structure is unlikely to occur [5-6]. These desirable features have been exploited in the past by other MEMS researchers who have incorporated parylene into various devices; for example, the use of parylene in a micromachined capillary electrophoresis stage is described in [7].

Thick resist structuring using an SU-8-epoxy-based resin originally developed by IBM and subsequently optimized for MEMS applications has been demonstrated in [3]. This epoxy resist is exposed using conventional UV lithography techniques and can easily provide high aspect ratio micromolds compatible with eletroplating techniques.. In the present work, parylene is used as a sacrificial layer between a first (primary) electroplated structure (originally created using a micromold of thick photoresist), and a second (secondary) electroplated structure, created around the primary in a self-aligned, self-molding process. After the parylene is removed, an air-gap, corresponding to the original parylene thickness, is created between the primary and secondary electroplated structures.

This basic process can be implemented in two different ways, depending on the desired relative motion between the first and second structures once the parylene is removed. The first process introduces a generic method to create overlapped structures able to move normal to the substrate; these are designated in this paper as vertical structures (Fig 1/a). The second demonstrates the possibility to create two structures electroplated on the same substrate, leading to motion parallel to the plane of the substrate, resulting in motion similar to that of micromechanical comb actuators; these are designated in this paper as horizontal structures (Fig 1/b)

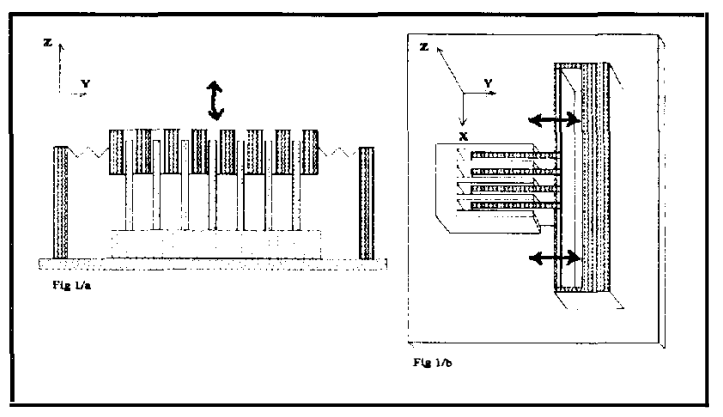

Figure 1: Examples of the two types of structures produced using this process. Fig. 1/a; side view of device capable of motion normal to the substrate (vertical device). Fig. 1/b; perspective view of device capable of motion parallel to the plane of the substrate (horizontal device). Both structures are formed using a self-aligned, self-molding process. 


\section{VERTICAL DEVICE FABRICATION}

The fabrication process for vertical devices consists of deposition of a primary structure through an epoxy mold, followed by conformal parylene coating, deposition of the secondary metal structure, and isotropic removal of the parylene mold. A description of the fabrication process is presented in Figure 2.

\section{A. Ni-Fe electroplated structures into $\mathrm{SU}-8$ micro-molds}

A metal seed layer system consisting of $50 \mathrm{~nm}$ of $\mathrm{Ti}$, an overlying electroplating seed layer of $150 \mathrm{~nm}$ of $\mathrm{Cu}$, and a final $50 \mathrm{~nm}$ of $\mathrm{Ti}$ is evaporated onto oxidized 3 inch $<111>$ silicon wafer substrates. Next, 40 microns of thick photosensitive epoxy Epon SU-8 prepared as described in [3] is spin-cast onto the metal system.. Using exposure and development guidelines described in [3], the resist is photolithographically patterned to create a mold through which the primary NiFe structures are then electroplated as described in [2] (Figure 2/a). A long $\mathrm{O} 2 / \mathrm{CHF} 4$ plasma etch is used to remove the mold (Figure $2 / \mathrm{b}$ ). A short ultrasonic cleaning step can be performed so that any residue still remaining at the bottom of each structure is removed.

\section{B. Conformal coating and second electroplating}

At this point, the sample is conformally coated with 5 microns of Parylene-C (Figure 2/c). An overlying $\mathrm{Ti} / \mathrm{Cu} / \mathrm{Ti}$ seed layer system similar to that described above is then deposited (Figure $2 / d)$; it is destined to provide a base onto which a second structure will be electroplated. This second structure is electrically isolated from the first one, due to the conformal coat of parylene. The secondary metallic structure is then electrodeposited in a self-aligned fashion, using the bottom and sidewalls of the primary structure as a mold (Figure $2 / \mathrm{e}$ ). The parylene is removed by a long isotropic plasma etch (Figure 2f). Figure 3/a shows a scanning electron micrograph of a released vertical structure, along with a schematic drawing (Figure 3/b) of the structures to better illustrate the micrograph.

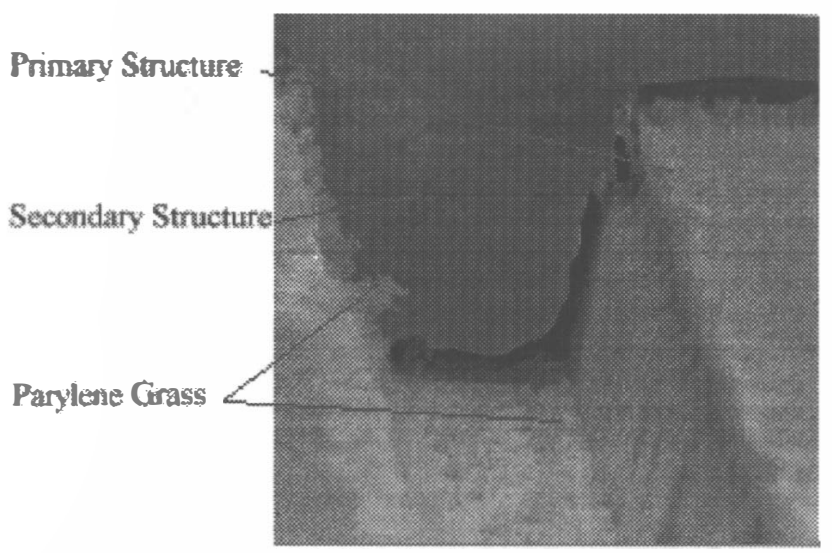

Figure 3a: Scanning electron micrograph (3/a) and schematic drawing (3/b) of a released vertical structure.

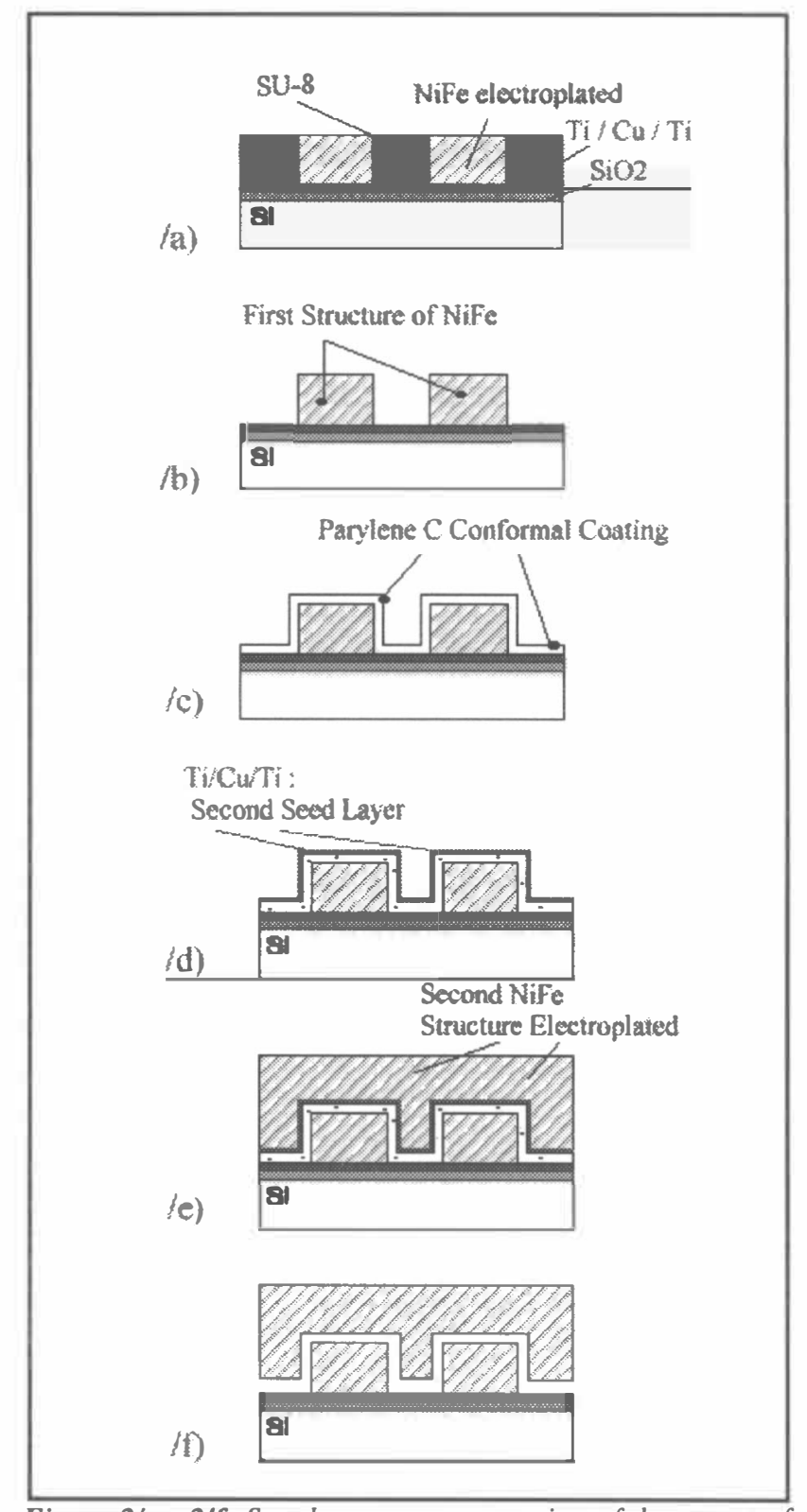

Figure 2/a - 2/f: Step-by-step representation of the process for fabrication of vertical devices.

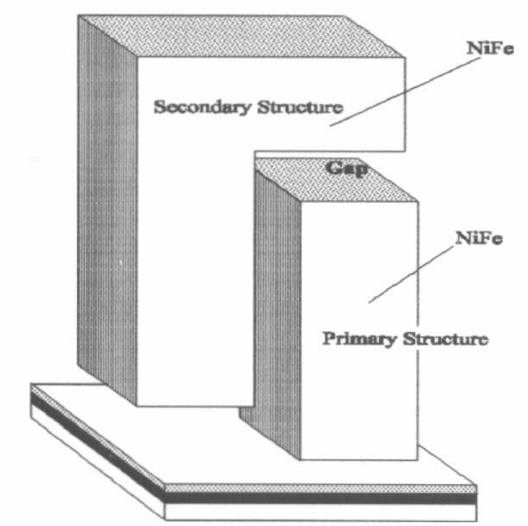

Figure $3 b$ : Schematic drawing (3/b) of a released vertical structure. 


\section{HORIZONTAL DEVICE FABRICATION}

\section{A. Ni-Fe electroplated structures into SU-8 micro-molds}

The same technique for the realization of $\mathrm{NiFe}$ electroplated structures into SU-8 micromolds presented in the vertical device fabrication section is utilized, with some change in process parameters since thicker epoxy films were utilized. An epoxy layer 230 microns in thickness is spin-cast, pre-baked, patterned, post-baked, and developed as described in [3]. The first electroplating step is performed (Fig 2/a). In order to reduce the surface tension of the bath and to avoid any bubbles remaining in these higher aspect ratio micromolds, $0.1 \mathrm{~g} / \mathrm{l}$ of a surfactant (sodium dodecyl sulfate) is added to the conventional $\mathrm{NiFe}$ galvanic bath. The surrounding epoxy is then removed using a dry etch similar to that used for the vertical structure fabrication described above.

\section{B. Conformal coating and second electroplating}

At this point, 28 microns of parylene $\mathrm{C}$ are deposited (Figure 4/a). An overlying $100 \mathrm{~nm}$ thick aluminum layer is sputter-deposited and patterned using conventional positive photoresist.. The aluminum mask must be wider than the previous NiFe structure by at least the thickness of the deposited parylene C itself (Figure $4 / b$ ). The role of this $\mathrm{Al}$ mask is to protect the parylene on the upper surface of each of the primary structures, as well as the regions near the tops of each of the parylene-C-coated sidewalls. Poor alignment during this step can result in etching of the parylene at the top corners of the primary structure, as shown in Figure 5, which would result in additional 'mushroom-like' plating on this exposed region when the secondary structure is electrodeposited. In some applications, this additional plating may be desirable; for example, to act as a retainer for the horizontal structures. A highly anisotropic reactive ion etching (RIE) step then removes the parylene exclusively at the bottom of each $\mathrm{NiFe}$ structure (Figure $4 / c$ ). The etch must be sufficiently anisotropic in order to not etch the side walls; this is achieved by performing the etch in a low pressure, high power environment. In some cases, small amounts of fluorine-containing species were added to the etch environment to reduce the problem of parylene 'grass' formation. The RIE step usually removes the Ti layer of the underlying seed layer $\mathrm{Ti} / \mathrm{Cu} / \mathrm{Ti}$ system deposited in part $(\mathrm{A})$. The exposed copper is used as a base for the next electroplating step, but it also can provide a useful visual test to determine when the parylene etch is complete

The parylene still present on the side walls of each structure will act as a spacer between the primary structure and the about-to-be-electrodeposited secondary NiFe structure. A second electroplating step is now performed (Figure 4/d) using the original seed layer, resulting in a secondary metal structure formed in a self-aligned fashion between the walls of the primary structure, but insulated from it by the parylene layer. A long isotropic plasma etch then removes the parylene sacrificial layer (Figure 4/e).

Figure 6/a shows a scanning electron micrograph of a released horizontal structure, along with a schematic drawing (Figure 6/b) of the structure to better illustrate the micrograph. There is some parylene still remaining between the primary and secondary microstructures, indicating that some further plasma etching is needed. The gap between the two structures is 24 microns and the final aspect ratio achieved in a first attempt is already better that 1 to 8 for the central secondary structure and better than 1 to 9 for the air gaps surrounding the second structure. It should be re-emphasized that the secondary structure is self-aligned with the primary since the primary structure has been used as a mold for forming the secondary structure.

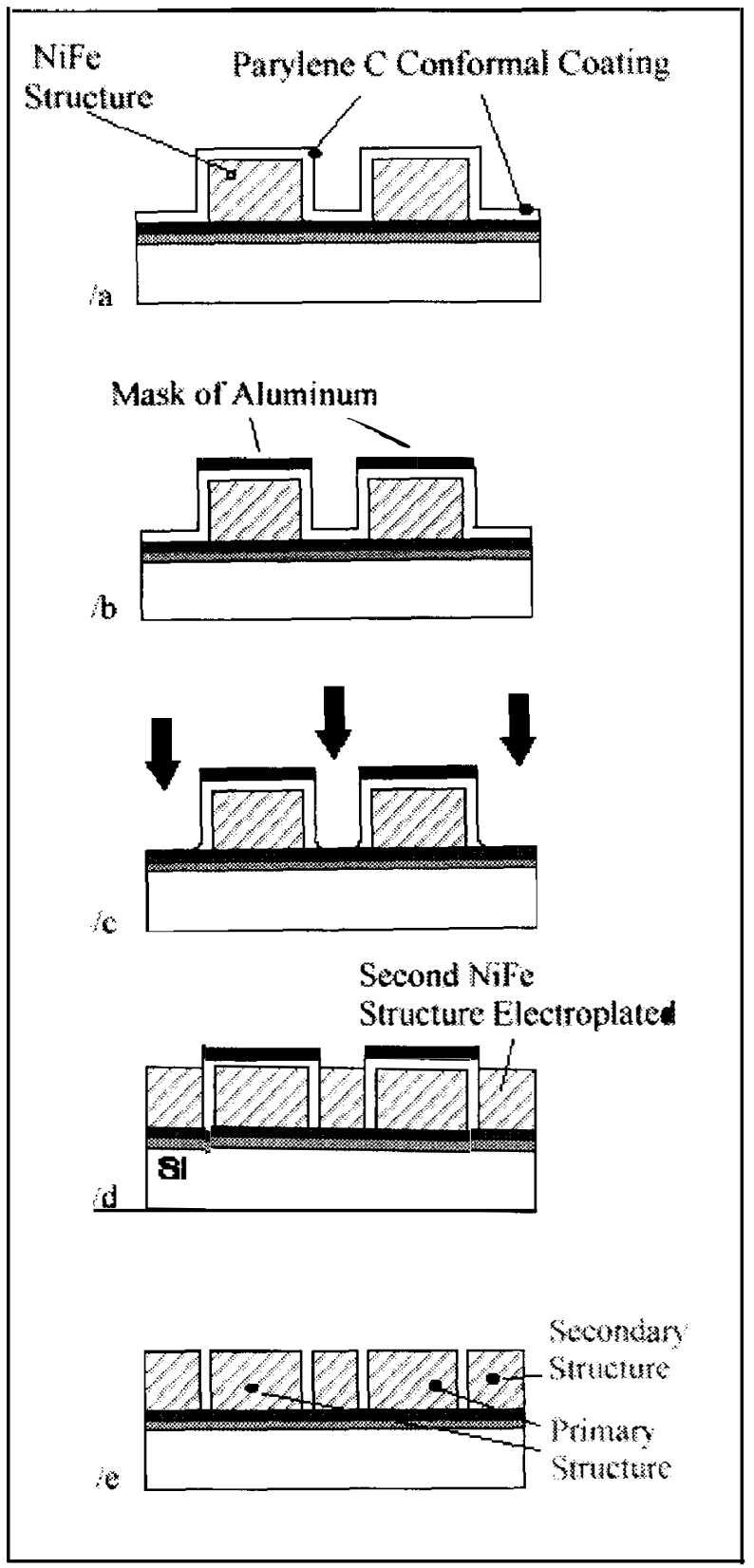

Figure 4/a - 4/e: Step-by-step representation of the process for fabrication of horizontal structures. 


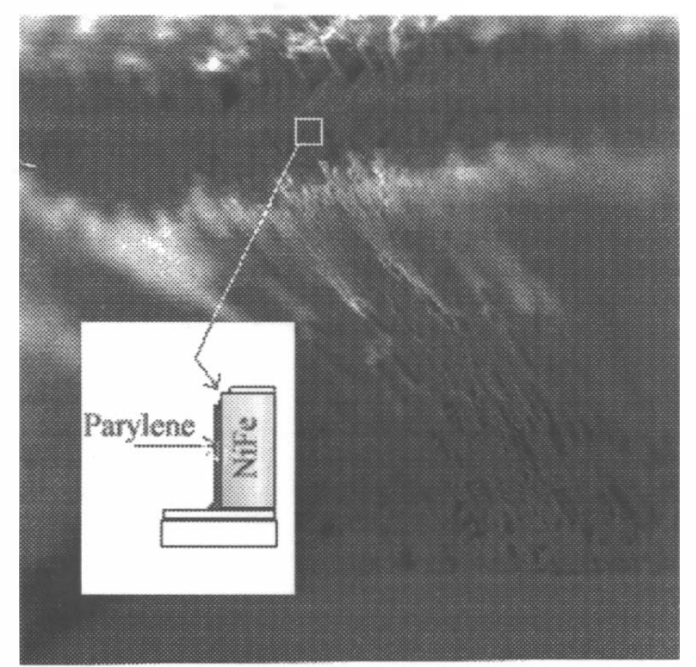

Figure 5: Scanning electron micrograph of a poorly-aligned structure, illustrating a gap between the parylene coated on the sidewalls and on the top of the primary structure.

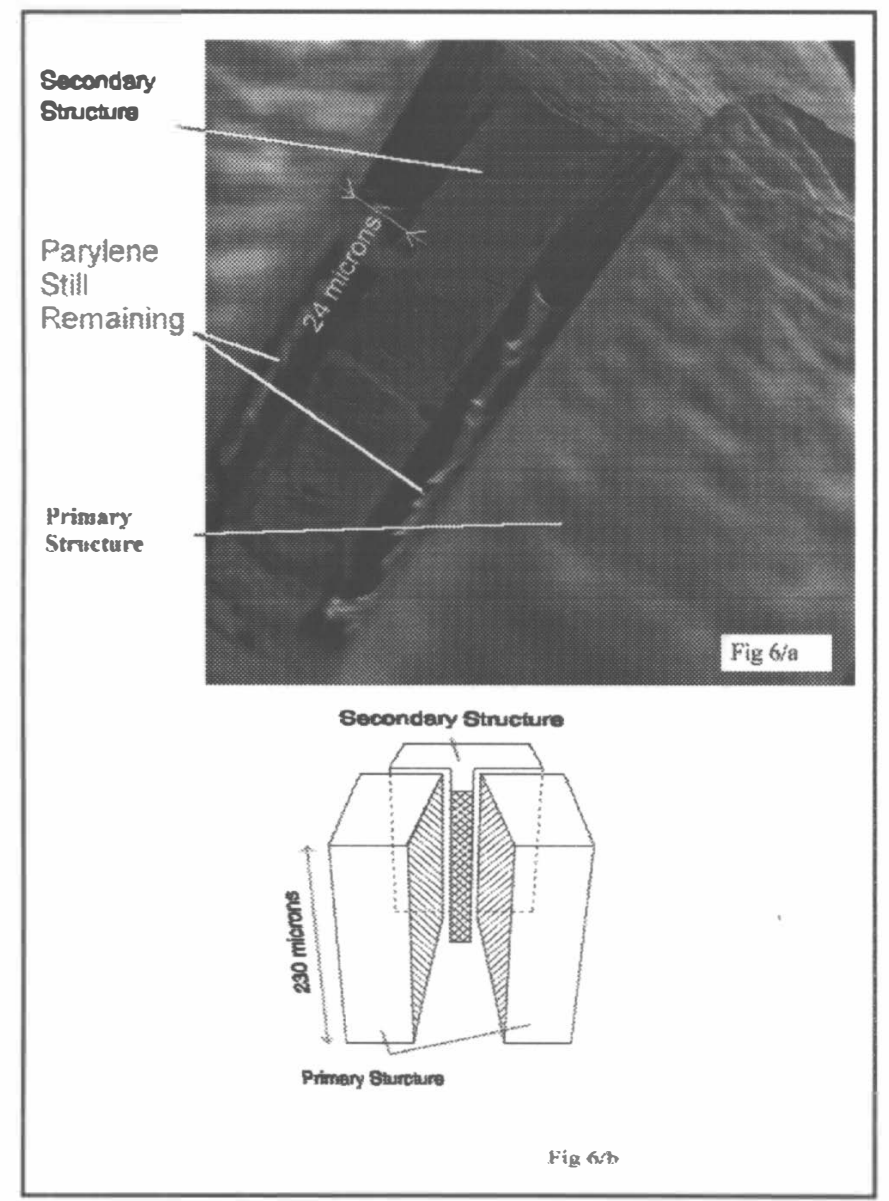

Figure 6: Scanning electron micrograph (6/a) and schematic drawing $(6 / b)$ of a released horizontal structure.

\section{CONCLUSIONS}

Two processes based on parylene conformal sacrificial layers have been presented for the realization of self-aligned and molded, vertical and horizontal released devices. Used in conjunction with high-aspect-ratio processing such as SU-8based electroplating of metallic structures, these parylene-based processes offer the potential to create extremely narrow gap structures with a variety of pertinent MEMS applications.

\section{REFERENCES}

[1] E.W. Becker, W. Ehrfeld, P. Hagmann, A. Maner, and D. Munchmeyer, "Fabrication of Microstructures with High Aspect Ratios and Great Structural Heights by Synchrotron Radiation, Lithography, Galvanoforming, and Plastic Moulding (LIGA process)", Microelectronic Eng., vol. 4, p. 35-56 (1986)

[2] A.B. Frazier and M.G. Allen, "Metallic Microstructures Fabricated Using Photosensitive Polyimide Electroplating Molds", Journal of Microelectromechanical Systems, vol. 2, no. 2, p. 87-94 (1993)

[3] M. Despont, H.Lorenz, N. Fahmi, P. Renaud and P. Vettiger, "High-Aspect-Ratio, Ultrathick, Negative-Tone NearUV Photoresist For MEMS Applications", Sensors and Actuators A (Physical), 1 Jan. 1998, vol.A64, n.1, pp.33-9

[4] A.B. Frazier and M.G Allen, "Uses of Electroplated Aluminum for the Development of Microstructures and Micromachining Processes", Journal of Microelectromechanical Systems, June 1997 vol.6, n², pp.91-8

[5] L. You, G.-R. Yang, C.I. Lang, P. Wu, J.A. Moore, J.F. McDonald, and T.M. Lu, "Vapor Deposition Of Parylene Films From Precursors”, Mat. Res. Soc. Symp. Proc. vol. 282, 1993, pp 593-598

[6] "Parylene For Electronics", available from Paratronix, Inc., Attleboro, MA

[7] J.R. Webster and C.H. Mastrangelo, "Large-Volume Integrated Capillary Electrophoresis Stage Fabricated Using Micromachining of Plastics on Silicon Substrates", Proc, Transducers '97, vol. 1, p. 503-506 (1997) 